\title{
Reports
}

\section{Regulatory Opportunities and Challenges in Commercialising Biobased Chemicals}

\author{
Lynn L Bergeson*
}

The $21^{\text {st }}$ Century has witnessed intense renewed interest in commercialising new biobased chemicals, defined generally to include chemicals that are derived from plants and other renewable materials. The Toxic Substances Control Act (TSCA) is the U.S. law that regulates industrial chemical substances, including biobased chemicals, used in applications other than food, drugs, cosmetics, and pesticides, or uses that are regulated by other federal authorities. TSCA was significantly amended in 2016 , and stakeholders need now more than ever to understand how TSCA applies to biobased chemicals to appreciate the implications of new TSCA on their commercial operations. Doing so will better assure uninterrupted business operations and consistent TSCA compliance.

\section{TSCA Overview}

TSCA authorizes the U.S. Environmental Protection Agency (EPA) to regulate 'chemical substances,' defined broadly to include 'any organic or inorganic substance of a particular molecular identity.' The term 'chemical substance' does not include pesticides, drugs, and food, which are regulated under other federal laws. A chemical substance for TSCA

DOI: $10.21552 / \mathrm{icrl} / 2019 / 1 / 6$

* Lynn L. Bergeson is the Managing Partner of Bergeson \& Campbell, P.C. (B\&C), a Washington, D.C. law firm focusing on conventional, nano, and biobased chemical, pesticide, and other specialty chemical product approval and regulation, environmental, health, and safety law, chemical product litigation, and associated business issues. For Correspondence: <lbergeson@lawbc.com>

1 The confidential portion of the Inventory can only be accessed in two ways. First, one could submit a PMN for the substance in question, because upon receipt of the PMN the first action by EPA will be to search the existing public and confidential inventories to determine whether a PMN is needed for the substance. Second, a confidential listing may be found by submitting a bona fide request to EPA, requesting whether the substance in question is present on the confidential Inventory. purposes includes plant or animal-derived substances and microbes used for TSCA purposes. TSCA is a complicated law with many interesting provisions and fascinating policy implications. The narrow focus here is on TSCA Section 8(b)(1) and Section 5 , as an understanding of these provisions is critical to understanding how TSCA applies to biobased substances of biological or microbial origin.

TSCA Section 8(b)(1) directs EPA to compile and keep current a list, commonly referred to as the 'TSCA Chemical Substance Inventory,' of each chemical substance that is domestically manufactured or imported into the U.S. The initial Inventory was developed from 1978 to 1979 using input from the chemical industry that allowed 'existing' chemical substances already in commerce to be 'grandfathered' onto the Inventory. These chemicals were included on the TSCA Inventory automatically, and were not subject to EPA review at the time of the listing. The Inventory is significant because if a chemical is not included on the Inventory, and is not otherwise exempt (eg used for research and development), the commercial production, distribution, and use of the substance is impermissible.

The TSCA Inventory is divided into two parts: the publicly accessible non-confidential portion that is readily accessed using the Chemical Abstracts Service (CAS) Registry Numbers (CASRN) for chemical substances; and a confidential portion in which only generic identities are available to the public while the specific identities are accessible only by EPA through its TSCA Inventory Master File. ${ }^{1}$

New chemical substances (any substance not listed on the TSCA Inventory) may be added to the TSCA Inventory via a process that involves submission of a Premanufacture Notification (PMN) or a Microbial Commercial Activity Notice (MCAN) for microbes, as described below. EPA typically reviews ap- 
proximately 1000 or so new chemical substances each year. Once EPA completes review of the new chemical and imposes any needed regulatory requirements necessary to abate potential unreasonable risk, the notifier is permitted to commence import or manufacture. The notifier must then submit a Notice of Commencement (NOC), receipt of which triggers EPA to add the chemical substance to the Inventory, transforming it into an existing chemical substance.

Adding chemicals to the Inventory has always been challenging, but it is more so today as a consequence of the 2016 TSCA amendments. These challenges are especially frustrating to entities wishing to use an expanded array of chemicals derived from sources not specifically listed in the TSCA Inventory. As discussed below, there seems to be a bias against biobased chemicals, despite the potential environmental and human health benefits.

TSCA Section 5 governs the manufacture in and import into the U.S. of chemical substances considered 'new.' Manufacturers (a term that includes importers) of chemical substances considered new must notify EPA of the new chemical substance through the submission of a PMN or MCAN. Unless a PMN exemption applies, ${ }^{2}$ a company must submit a completed PMN form to EPA at least 90 days before commencing the manufacture or import of a new chemical substance. Although the statutory minimum is 90 days, in practice a company should allow more time for PMN or MCAN review.

Under revised TSCA Section 5(a)(3), EPA is required to review all new chemical notifications (both PMNs for conventional chemicals and MCANs for microorganisms) and Significant New Use (SNU) notifications, make one of three determinations, and take required actions, as outlined below, depending upon the determination. In evaluating whether an 'unreasonable risk' is presented by such cases, EPA is required to consider potentially exposed or susceptible populations identified as relevant and, in some cases, this includes the concept of 'under the conditions of use.' EPA is also required to undertake a staged review focusing initially on the determination and then on the action required given the determination made. As is true generally under new TSCA, as part of this staged process, EPA is disallowed from considering cost or other non-risk factors in determining whether a chemical presents an unreasonable risk.
This approach to new chemical review represents a fundamental departure from old TSCA. Previously, new chemical innovators submitted a notice under TSCA Section 5 and if EPA declined to take action in 90 days, the submitter could commence chemical production or import followed by the submission of an NOC. Under new TSCA, this passive approach has been replaced by an active one and an EPA determination is required along with any needed regulatory actions as a predicate to chemical production for commercial purposes.

In satisfying the requirement that EPA make a determination and take required actions on all new chemicals and SNUs, there are three alternative determinations available to EPA under new TSCA. First, EPA can determine that the new chemical or SNU presents an unreasonable risk of injury to health or the environment, in which case, EPA is required to regulate under Section $5(\mathrm{f})$ and must then also promulgate a Significant New Use Rule (SNUR) or explain why not.

The second alternative consists of a series of 'or' statements that reflect some uncertainty in the risk. EPA can determine the information available on the chemical is insufficient to permit a reasoned evaluation of the health or environmental effects of the chemical, or, in the absence of sufficient information, the substance may present an unreasonable risk, or

2 There are exemptions from the requirement to submit a PMN Exemptions are either 'self-executing' or require prior EPA approval. Self-executing exemptions are those that take effect once an entity determines that the exemption applies, and the new chemical substance can be manufactured in the U.S. without the need for a PMN, provided the company complies with any recordkeeping or other applicable requirements for the particular exemption. Self-executing PMN exemptions include the exemption for chemical substances having no separate commercial purpose, the polymer exemption, and the research and development (R\&D) exemption. Other exemptions from the PMN requirement require prior EPA approval. Entities must submit, and EPA must approve, an exemption application before the entity can commence manufacture of the new chemical, subject to compliance with any recordkeeping or other applicable requirements. PMN exemptions that require prior EPA approval include the low volume exemption (LVE), the low release and low exposure exemption (LoREX), and the test marketing exemption (TME). Eligibility for an LVE is based on the manufacture of a new chemical in quantities of 10,000 kilograms or less per year, while eligibility for a LOREX is based on meeting several regulatory criteria for 'low' release and exposure throughout the manufacture, processing, distribution, use, and disposal of the chemical (40 C F R Sections 723.50(a) and (c)). One kilogram is equivalent to 2.2 pounds. Once EPA notifies an applicant that its LVE or LoREX application has been granted, or if the 30-day review period expires without notice from EPA, manufacture or import of the chemical substance may commence, consistent with the terms of the exemption. 
Table 1

\begin{tabular}{|l|l|}
\hline Soybean-based supply chain & Corn-based supply chain \\
\hline soybean oil & corn oil \\
\hline fatty acids, soya & fatty acids, corn-oil \\
\hline fatty acids, soy, ethoxylated & fatty acids, corn-oil, ethoxylated \\
\hline
\end{tabular}

that the substance will be produced in substantial quantities and it either enters or may be anticipated to enter the environment in substantial quantities, or that there is or may be significant or substantial human exposure. If any of these determinations is satisfied, EPA is required to issue an order under Section 5(e) and either to implement a SNUR or explain why it is not taking this step.

The third determination EPA can make is that the new chemical or SNU is not likely to present an unreasonable risk, in which case, the notice submitter may commence manufacture/processing forthwith once the determination has been made, notwithstanding any remaining portion of the applicable review period. EPA is also required to publish a statement of its finding. This determination and its statement are not legally reviewable. EPA makes all such notices available on its website and publishes them in the Federal Register.

When preparing a PMN or MCAN submission, it is critical that attention be paid to the substance identity, as it can affect the TSCA regulatory status of the substance and its downstream products as new or existing substances. For substances with a single, well-defined chemical structure, such as ethanol and acetic acid, the naming conventions and TSCA Inventory search are relatively straightforward. The precise determination of the chemical identity and TSCA Inventory status of a substance lacking a definitive molecular formula or structural diagram can be more complicated. These less-defined substances, referred to as Class II chemicals, include 'unknown or variable composition, complex reaction products, or biological materials' (UVCB). UVCB substances are often identified by the 'source' and chemical processes used during manufacturing. The sourcebased nomenclature system results in multiple nomenclature listings for nearly equivalent chemi- cal substances that are derived from different sources.

For example, soybean, canola, and sunflower oil are each listed separately on the TSCA Inventory and a company cannot rely on any of these listings to manufacture or import an oil from another source, even if such substances have indistinguishable compositions. Companies should also be aware that the Class II nomenclature system propagates through the supply chain. For example, if each of the soybean, canola, and sunflower oils are converted to fatty acid methyl esters (FAME), the individual FAMEs are listed separately (eg, fatty acid, sunflower, Me ester). This so-called 'source-based' system has significant business implications for supply chains that rely on biobased substances and other stakeholders. Table 1 provides an example of source-based identities in a supply chain.

The identities for these two supply chains are not interchangeable, even though the compositions are very similar and there is no discernable difference in the risk to health or the environment.

In 1979, EPA attempted to streamline the Class II nomenclature system by developing a source-agnostic system with the help of the Soap and Detergent Association (SDA) (now the American Cleaning Institute). The SDA nomenclature system, which is based on substance type and alkyl range rather than source and processing, allows for significant feedstock and operational flexibility by drawing equivalence between chemical substances produced from 35 natural sources of fats and oils and their petroleum-based counterparts, but limits eligibility to these sources. Note that the SDA policy states 'Alkyl groups derived from other natural sources are not covered by this system.' EPA interprets this statement to mean that sources that are not listed are not eligible to use the SDA nomenclature. 
Sources not eligible for SDA nomenclature include: tall oil, jatropha oil, camelina oil, waste oils and grease, algal oils, and oils produced by Genetically Modified Organisms (GMO). This means that the manufacturer of a novel source chemical substance (eg, algal oil) is required under TSCA to submit to EPA a chemical notification under TSCA Section 5 to add the substance to the TSCA Inventory before commercialising the material. Even more consequential is the fact that the manufacturer's customers may also be required to submit PMNs for downstream UVCB substances that are produced from it, such as free fatty acids and biodiesel. The time and business planning that it takes to accomplish these inconvenient realities cannot be over-emphasized, especially now that new TSCA requires a more disciplined and time-consuming review of new chemical notifications.

\section{SDA Nomenclature Source}

New TSCA provides EPA with authority to recognize multiple listings of a substance listed on the Inventory as a single substance. EPA is not mandated to exercise this authority, but discussions between industry and EPA are underway to achieve this goal. To support the efficient commercialisation of biobased products, the microbial chemical industry would be wise to engage with EPA to ensure that consideration is given to a wider range of sources, not just the ones that were available in 1979 (See Figure 1). Expansion along these lines would facilitate operational flexibility and level the playing field for new product entrants that are based on sources that fall outside the listed 35 natural sources, but have compositions that are largely, if not entirely, the same as those existing natural sources.

Naturally occurring substances are exempt from Section 5 reporting since EPA considers such substances to be automatically listed on the TSCA Inventory. This category of substances is important to stakeholders for reasons that need no explanation. EPA has defined 'naturally occurring' substances quite narrowly, however, and care should be taken to understand its scope for TSCA purposes. Microorganisms that do not contain genetic material from organisms of a different taxonomic genus may be considered naturally occurring, whereas intergeneric microorganisms are not naturally occurring. Im- portantly also, the processing of a microbe or other biobased substance beyond the discrete methods described in the definition of naturally occurring substances (eg, manual or mechanical processing) likely results in a substance that EPA would not consider naturally occurring and thus exempt.

Depending on their end use, intergeneric microorganisms, feedstocks, intermediates, byproducts, enzymes, and other catalysts may all be reportable under TSCA. Furthermore, companies that rely on byproducts or waste as a feedstock should engage with their supplier regarding the TSCA status of the feedstock to avoid undue supply delays. For example, yellow grease (waste glycerides from kitchen uses) is listed on the TSCA Inventory, so it may be used as a feedstock for growing microbes for a non-exempt TSCA purpose. Brown grease (waste glycerides from sewage treatment), however, is not listed on the Inventory, so EPA would likely be of the view that a bioeconomy company could not use brown grease as a feedstock for a commercial purpose regulated under TSCA without first submitting a PMN.

The biobased chemical community may also be interested in some of the more general provisions that were introduced as part of new TSCA. For instance, Section 14 now requires that companies substantiate many confidential business information (CBI) claims at the time the confidential information is submitted to EPA. The substantiation process takes time. Careful consideration and a rigorous process must be part of the business process to ensure that confidential information is protected.

New TSCA also modified Section 14 to make it explicit that some information is not protected from disclosure, including mixed confidential and nonconfidential information, general (high-level) descriptions of the manufacturing, and/or processing and aggregated production volumes. Companies should also be aware that health and safety studies cannot be protected as CBI (although the identity of the test substances may be confidential, if justified). Additionally, new TSCA requires EPA to consult with the Small Business Administration (SBA) to review the adequacy of the current standards for small manufacturers and determine whether a revision of the definition of a 'small' business is warranted, which EPA has done. EPA's October 17, 2018, final rule on TSCA user fees adjusted the small business size standard. EPA ultimately decided to apply an employeebased definition modeled after the SBA's approach. 


\begin{tabular}{|c|c|c|c|}
\hline \multicolumn{2}{|c|}{ Vegetable } & \multirow{2}{*}{$\underline{\text { Animal }}$} & \multirow{2}{*}{$\begin{array}{l}\text { Marine } \\
\text { Herring }\end{array}$} \\
\hline Avocado & Peanut & & \\
\hline Babassu & Rapeseed & Lard & Menhaden \\
\hline Castor & Rice Bran & Neatsfoot & Salmon \\
\hline Coconut & Safflower & Poultry & Sardine \\
\hline Corn & Safflower & Tallow & Sperm Body \\
\hline Cottonseed & (high oleic) & & (whale) \\
\hline Crambe & Sesame & & Sperm Head \\
\hline Linseed & Sorghum & & (whale) \\
\hline Olive & Soybean & & Whale \\
\hline Oiticica & Sunf lower & & \\
\hline Palm & Tung & & \\
\hline Palm-kerne1 & Wheat Germ & & \\
\hline \multicolumn{4}{|c|}{$\begin{array}{l}\text { These sources provide both saturated and unsaturated alkyl groups; } \\
\text { castor oil provides a } C_{1} \text { hydroxy substituted alkyl group. Alkyl groups } \\
\text { derived from other natural sources are not covered by this procedure. }\end{array}$} \\
\hline
\end{tabular}

Figure 1: (U.S. EPA, 1978)

\section{What Should You Do To Commercialise Biobased Chemicals?}

The full implications of new TSCA will become clearer as EPA fully implements its new authorities and EPA's deployments of these authorities are judicially refined. It is crucial that biobased stakeholders be familiar with TSCA's statutory provisions and EPA's implementation of them, and engage meaningfully and robustly in implementation activities that impact the development, regulation, and commercialisation of chemical products. A thorough understanding of EPA's approach to chemical regulation is central to avoiding commercial disruptions and operation delays, competitive imbalances, and potential assertions of noncompliance.

Companies are also encouraged to develop a strong compliance program, to consider the regulatory timeline when formulating business plans, and to seek assistance from experts in the regulatory and legal fields regarding the preparation and review of EPA submissions. Innovators should develop strong, positive relationships with regulators based on trust and clear and open communication. Regulators are a critically important component of the stakeholder community and are often under-appreciated as one of a business' strongest supporters. While EPA may recognize and be receptive to the benefits of animal and plant-based products, it is still required to review and regulate such chemical substance if it determines that the substance may present unreasonable risk during the review process. Engaging with EPA early in the process to understand any potential concerns, how those concerns can be addressed, and how a product's pollution prevention attributes provide essential value to the economy may help companies avoid significant regulatory issues and potentially costly business delays down the road.

\section{Tips to Ensure Commercialise Success}

Below are a few tips to help ensure consistent compliance and predictable business operation:

\section{Ensure TSCA Compliance is a Core Element of the Business Plan}

The first tip we offer is to know TSCA requirements, understand the regulatory responsibilities, and be prepared to meet both the requirements and the responsibilities as a part of a business development plan. TSCA provisions should not be collateral to the business plan; they must be a core element embedded in the planning process. A good command of TSCA will decrease the likelihood of a major, unanticipated disruption to the commercialisation timeline due, for example, to late recognition of the need for a PMN, MCAN, or other significant pre-market approval issue. 


\section{Understand the Relevance of Chemical Naming Conventions}

It is critically important to recognize and understand the importance of how a chemical substance is named and identified for TSCA purposes, and how that identity is determinative to a manufacturer's responsibilities. There is both art and science involved in naming a TSCA chemical. It is important to understand the relevance of naming conventions to the manufacturing process. If this core competency does not exist within the company's staff, find competent professionals who can guide this important process. As arcane as this point may seem, it could make a critical difference in the timing of the commercialisation process.

\section{Know the Fundamentals of the TSCA Review Process}

A basic understanding of EPA's review process and regulatory approach is essential. While EPA works off of the information included in the PMN/MCAN, it also considers information on other 'related' cases, applies quantitative structure-activity relationships ((Q)SAR) analysis when hazard test data are not available, and, inter alia, will use assumptions about potential exposures and releases if information is not provided in the notification. EPA also has a number of policy drivers that can affect new chemicals, including its use of 'categories' of PMNs, the persistent, bioaccumulative, and toxic (PBT) policy, and the 'exposure-based' policy for new chemicals. ${ }^{3}$ It is useful to understand and be able to anticipate (and where possible, avoid) the potential effect of these policy drivers.

\section{Consider Testing in Advance of PMN/MCAN Notification}

If EPA is likely to impose testing requirements on a biobased new chemical, consider the benefits of either doing the testing in advance of the notification (and thus avoiding that issue), or, if future commercialisation plans involve additional structurally similar new chemicals, whether it might make sense to develop a testing strategy that would attempt to encompass and account for the range of new chemicals likely to be introduced. While such a strategy could be implemented by a single company, if other firms are known to be active in this area of new chemical development, there might be significant cost saving and advocacy opportunities for organizing consortia to share the costs and responsibility of testing. EPA is also more likely to be receptive to a consortium's regulatory advocacy, as opposed to a single company's efforts to influence new chemicals policy. Any testing strategy should include consideration of future market opportunities (eg, the European Union), so that registration requirements imposed by other countries can be addressed or satisfied with the testing performed to support a TSCA notification.

\section{Work with EPA}

Regardless of the approach taken, it is always wise to consult with EPA before embarking on chemicalspecific testing or developing and implementing a testing strategy. This will ensure an understanding of EPA's views on and obtain its receptivity to the approach proposed as well as addressing, in advance, any of EPA's concerns about adequacy of the test method.

\section{Conclusion}

Faithful adherence to a business plan informed by a keen understanding of TSCA and commitment to the tips noted above will help ensure commercial success. There is considerable upside in biobased chemical development as the public rewards alternative to

3 A thorough review of these policies is beyond the scope of this article. It is important to recognize, however, that EPA's chemical management program and TSCA review process is premised upon a number of important policies. For example, in 1999, EPA issued its final policy statement on a category of PBT chemicals. The policy statement reflects EPA's policy regarding PBT chemicals, and advises industry about EPA's regulatory approach for chemicals meeting the criteria of a PBT chemical. Another important policy is EPA's approach to chemical categories. EPA groups chemicals with shared chemical and toxicological properties into categories. According to EPA, this enables PMN submitters and EPA reviewers to benefit from collected data and 'past decisional precedents' in the PMN review process. What this means in practice is that if PMN submitters are unaware of the chemical categories approach, essential elements of EPA's approach to the review of a particular PMN submission may be overlooked in the preparation of the PMN and critical missteps could compromise the success of the PMN, resulting in lengthy delays or fatal mistakes. 
petroleum-based chemistries. TSCA applies to both types of chemicals, however, and mastering TSCA is critical to commercial success. 